\title{
Corela
}

Cognition, représentation, langage

HS-31 | 2020

Métalinguistiques.

\section{Hétérogénéité des pratiques et du lexique métalinguistiques en classe de FLE/S}

\section{Angélique Masset-Martin}

\section{(2) OpenEdition}

\section{Journals}

Édition électronique

URL : http://journals.openedition.org/corela/11653

DOI : 10.4000/corela. 11653

ISSN : 1638-573X

Éditeur

Cercle linguistique du Centre et de l'Ouest - CerLICO

Référence électronique

Angélique Masset-Martin, « Hétérogénéité des pratiques et du lexique métalinguistiques en classe de FLE/S », Corela [En ligne], HS-31 | 2020, mis en ligne le 02 juillet 2020, consulté le 03 juillet 2020. URL http://journals.openedition.org/corela/11653 ; DOI : https://doi.org/10.4000/corela.11653

Ce document a été généré automatiquement le 3 juillet 2020

\section{(c) (i) (2) (2)}

Corela - cognition, représentation, langage est mis à disposition selon les termes de la licence Creative Commons Attribution - Pas d'Utilisation Commerciale - Partage dans les Mêmes Conditions 4.0 International. 


\title{
Hétérogénéité des pratiques et du lexique métalinguistiques en classe de FLE/S
}

\author{
Angélique Masset-Martin
}

\section{Introduction}

1 Notre article s'inscrit dans le champ de la didactique des langues et plus précisément de la didactique du français langue étrangère (FLE). Le FLE a plusieurs déclinaisons, suivant le public auquel l'enseignement du français s'adresse. Dans notre cas, il s'agit d'enfants et de jeunes adolescents allophones scolarisés en France, le français enseigné est donc qualifié de "langue seconde » (FLS) dans la mesure où l'enseignement/ apprentissage se fait en contexte homoglotte ${ }^{1}$. Notre public est hétérogène à de nombreux niveaux : langue maternelle, scolarisation antérieure, pays d'origine, etc.

2 En classe de langue, et cela n'est guère surprenant puisque la langue est l'objet de tous les propos ou presque, le métalinguistique est omniprésent. Des heures d'observations et d'enregistrements de cours tenus dans différents types de classes accueillant ces élèves allophones, nous ont permis d'élaborer un corpus ${ }^{2}$, de l'analyser, et de mettre au jour un certain nombre de caractéristiques liées au métalinguistique. Nous en retiendrons deux dans le cadre de cet article.

3 La première caractéristique concerne l'hétérogénéité de ce que nous appelons les «pratiques » métalinguistiques. Quelles sont-elles? Quelles formes prennent-elles dans nos classes de FLE/S ? (partie 1)

4 La deuxième a trait au lexique métalinguistique. En quoi ce dernier est-il hétérogène ? Comment peut-on le qualifier? Le classifier? (partie 2) 


\section{Hétérogénéité des pratiques métalinguistiques}

5 Nous entendons ici par "pratiques" les moments de la classe où la langue est spécifiquement l'objet des discours tenus, des interactions, interactions que l'on peut également qualifier de métalinguistiques. Trévise (1997: 48) écrit que « [...] l'interaction métalinguistique entre enseignants et apprenants facilite l'apprentissage d'une langue maternelle ou étrangère en milieu scolaire [...] » et c'est exactement ce que nous avons $\mathrm{pu}$ observer. Ce sont ces interactions portant sur la langue qui permettent aux apprenants d'avancer dans leur apprentissage, et il est donc important de leur fournir toutes les occasions de s'exprimer (verbalement ou non). En effet, les interactions, qu'elles soient métalinguistiques ou didactiques d'ailleurs, sont indispensables dans des classes de langue seconde telles que celles que nous avons observées et doivent être sollicitées, afin que les élèves s'approprient cette langue qui fait partie de leur environnement scolaire ou extra-scolaire.

6 Les pratiques métalinguistiques observées, enregistrées et recueillies dans les classes sus-mentionnées sont hétérogènes dans la mesure où, d'une part, elles prennent des formes diverses et où, d'autre part, elles ne sont pas toutes circonscrites à la classe de langue (si l'on excepte les moments d'explication du fonctionnement de la langue ${ }^{3}$ ) puisqu'elles sont observables également dans le quotidien des élèves, en dehors de la classe, dans des situations de communication courante entre des interlocuteurs qui parlent, ou non, la même langue. Il existe une sorte de continuum des pratiques métalinguistiques depuis les plus spécifiques à la classe de langue, à celles qui le sont moins (voire pas du tout), et c'est en suivant ce continuum que nous allons présenter brièvement celles qui nous semblent les plus représentatives. Ceci nous intéresse en ce que ce continuum se retrouve dans le métalexique, depuis le plus « savant » (verbe, COD) jusqu'à celui très fréquent dans les discours ordinaires (dire, s'appeler). Or, nous pensons qu'une prise de conscience par les enseignants de cette perméabilité des frontières entre les différents discours pourrait amener à un usage plus raisonné de la terminologie mise à leur disposition dans les textes officiels ${ }^{4}$.

\subsection{L'explication linguistique}

7 Sans surprise, (tenter d')élucider le fonctionnement de la langue est une activité très répandue dans notre contexte de scolarisation en France. Cette explication s'accompagne le plus souvent d'une terminologie bien précise, "spécialisée ", commune à l'ensemble des enseignants puisque c'est celle que l'on retrouve dans les textes officiels.

Pour les non natifs, cette terminologie est bien souvent inconnue, a fortiori pour les élèves n'ayant jamais été scolarisés auparavant ou pour ceux venant de pays où la focalisation sur la grammaire que l'on trouve en France n'existe pas (pays anglo-saxons notamment). Dans ce cas, nous avons parfois observé chez les enseignants une utilisation métaphorique de cette terminologie, voire même pour les métatermes qui s'y prêtent, des définitions 5 .

9 Dans notre corpus nous avons relevé de nombreux moments consacrés à la grammaire menée dans une démarche assez classique, et par conséquent explicite, au sens où le discours tenu par l'enseignant comporte des termes bien spécifiques qu'il emploie dans l'énoncé d'une règle ${ }^{6}$, que celle-ci soit fournie avant exemples et exercices (démarche 
déductive), ou après (démarche inductive), ou qu'elle soit donnée à construire par les apprenants (ce qui est communément nommé « conceptualisation ${ }^{7}$ » en didactique des langues étrangères).

10 Cependant, avec le public des plus jeunes, à l'école élémentaire, et aussi avec les plus âgés dans certains $\operatorname{cas}^{8}$, l'explication de l'organisation de la langue peut se faire sans utilisation de mots métalinguistiques. Par exemple, monsieur E. (CLIN), lors d'une séance qu'il avait prévu de consacrer à la localisation, l'a organisée toute entière autour des déplacements des élèves, de leur position dans la classe, de la position de divers objets qu'il a fallu déplacer, etc. Ceci en répétant à de nombreuses reprises les notions nécessaires ("à côté de ", "devant ", "derrière », etc.), en organisant des jeux où "dans ", « autour », etc. doivent être maitrisés, en insistant sur ces lexies, le tout sans jamais expliquer ni comment s'appellent ces dernières ni comment elles fonctionnent. Pourtant, on se trouve toujours face à une pratique métalinguistique, la langue reste l'objet des échanges tenus dans la classe ${ }^{9}$ mais l'enseignement/apprentissage se fait sans règle, sans terminologie, sans « cours de grammaire ». Dans ce type de démarche, le but est de faire acquérir des structures par l'expérience, « mine de rien » pourrait-on dire. Cette démarche est donc métalinguistique pragmatiquement, les activités liées le sont aussi (reformulations, manipulations, répétitions) sans l'être dans la forme c'est-àdire sans terminologie métalinguistique.

\subsection{Les corrections / appréciations}

11 Le rôle de l'enseignant en classe de langue étrangère implique que ce dernier intervienne sur les erreurs de ses élèves, c'est pourquoi les corrections sont omniprésentes en classe de langue. La correction est pour nous une pratique on ne peut plus métalinguistique puisque la langue en est l'objet, et qu'il s'agit d'un « retour » sur un énoncé (oral ou écrit). Ces corrections se font le plus souvent à l'initiative de l'enseignant, qui signale l'erreur :

12 - de manière plus ou moins explicite: "non, ce n'est pas correct» (ex.1), « tu ne prononces pas bien/mal» (ex. 2) ou le plus souvent « on (ne) dit pas » (ex. 3) :

Ex. $1^{10}$

$<$ P> est-ce que c'est correct?

$<$ Plus. Elèves $>$ nnon\} \{c'est faux\}

$<$ P chut non ce n'est pas correct c'est faux voyons Maran

$<\mathrm{M}>$ il y a trois personnages

Ex.2

$<$ P e elle est Marocaine tu prononces mal Marocaine arrête avec ton cahier tu vas

me le salir Marocaine / quelle est sa nationalité ?

$<\mathrm{E}^{*}>$ il est [maroken]

$<$ P> il est Marocain Marocain ${ }^{11} /$ quelle est sa nationalité ?

Ex.3

$<$ A2 $>$ il y a un frère et mon sœur

$<\mathrm{P}>$ on dit pas il y a j'ai

$<A 2>$ j'ai un frère et un ma sœur

$<$ P> et une sœur pas un ma sœur d'accord ? [...]

13 - par la simple répétition de l'énoncé fautif sous forme de question, ou simple « non» que l'élève doit interpréter comme étant une invitation à se corriger :

$<$ I $>$ euh dix phrases

$<$ P recommence 


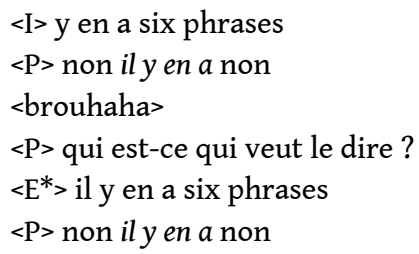

14 - par un commentaire métalinguistique du type « ce n'est pas français » :

$<\mathrm{P}>$ alors dans la deuxième page on voit que monsieur Mime

$<\mathrm{E}>$ il dormir

$<$ P $>$ il dormir c'est pas français

$<$ E $>$ il dort

15 - directement, sans indice clair, afin sans doute de ne pas interrompre la production de l'élève :

$<\mathrm{H}>$ Edina aujourd'hui pas vient

$<$ P $>$ non aujourd'hui Edina ne vient pas elle ne vient plus Edina elle vient avec les grands maintenant [...]

16 Les corrections/appréciations négatives sont le fait de l'enseignant dans la plupart des cas, mais elles peuvent aussi venir des apprenants, sur leur propre production (autocorrection) ou sur celle d'un autre apprenant (hétérocorrection). Notons que les apprenants calquent leur discours correctif sur celui de l'enseignant, et que par conséquent, on peut trouver des remarques telles que «il a fait une faute d'orthographe » de la part d'apprenants même débutants en français.

17 L'enseignant peut également approuver une production, mais dans ce cas, son assentiment est le plus souvent donné très rapidement à l'aide d'un simple « oui », ou d'un geste, même si on peut observer des appréciations positives plus élaborées (« c'est très bien »).

On le voit, la présence de mots métalinguistiques n'est pas nécessaire pour conclure au caractère métalinguistique de cette pratique particulièrement attestée dans notre corpus (cf. exemples). Leur absence peut toutefois être source d'ambiguïté, l'élève ne comprenant pas ce qui ne va pas : comme les erreurs relèvent de la forme ou du sens, quand l'intervention de l'enseignant est trop vague, les élèves ne savent pas quoi corriger :

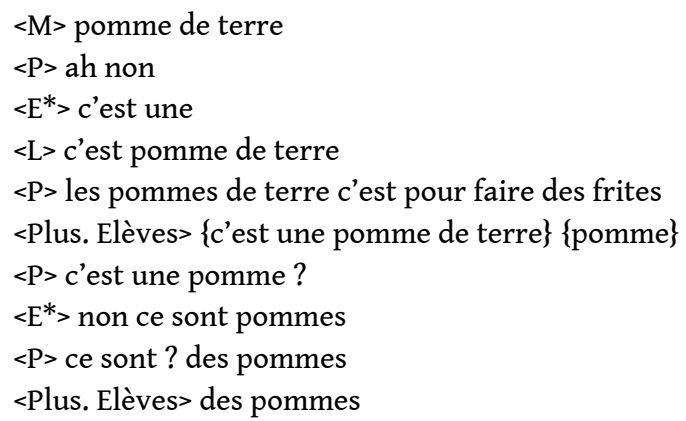

Dans cet extrait, on a un commentaire métalinguistique sans reprise de l'énoncé («ah non »). Il arrive alors que les élèves pensent que ce soit en raison d'une erreur sur la forme (puisque ce sont celles qui sont le plus souvent pointées). Ainsi un élève donne "c'est une », point qui fait l'objet de l'étude ${ }^{12}$, alors que l'enseignant refuse la réponse car elle ne correspond pas à la réalité. Il attend en effet pomme d'où «ah non » mal compris et complété par la réplique (« les pommes de terre c'est pour faire des frites»). 
Enfin, très souvent, la correction sert de point de départ à une explication de grammaire, notamment avec les élèves de collège (ce que nous appelons des "digressions métalinguistiques» qui rajoutent au constat que tout ou presque est métalinguistique en classe de langue).

\subsection{Les dénominations et définitions}

21 Une troisième pratique, extrêmement répandue dans les UPE2A observées et que l'on peut retrouver dans les interactions quotidiennes, en particulier lors de l'acquisition par le jeune enfant de sa langue maternelle ${ }^{13}$, est celle qui consiste à définir un mot inconnu, ou à nommer, l'accroissement du lexique de l'élève étant un objectif primordial. Toute occasion est saisie pour introduire des mots nouveaux et/ou leur(s) signification(s).

22 "Comment ça s'appelle?» (dénomination) et «qu'est-ce que ça veut dire?» (définition) sont deux des questions les plus fréquemment posées que ce soit par les enseignants ou par les élèves. Ces questions sont métalinguistiques puisqu'elles comportent des mots métalinguistiques (dire, s'appeler). Pourtant, une autre question, qui attend la même réponse, qui a donc la même fonction métalinguistique et qui pourtant ne comporte pas de métaterme est le «c'est quoi...»; «qu'est-ce que c'est... ? » suivie de l'autonyme ${ }^{14}$ (« qu'est-ce que c'est que entourer?») ou pas quand le référent peut être montré ("c'est quoi ça monsieur?»). La dénomination peut donc concerner des référents présents dans la classe, et la réponse donnée à « comment ça s'appelle ça? » ou "c'est quoi ça? » peut n'être que le nom de l'objet en question (et donc toute métalangue est absente). Si l'objet n'est pas là " physiquement» dans la classe, l'enseignant recourt aux photos, dessins, etc. ou, de nouveau, à la définition, voire à la traduction :

$<\mathrm{P}>$ les oiseaux mangent des graines oiseau un oiseau / des graines c'est des petits_ des petites choses que mangent les oiseaux dis-lui traduis-lui ${ }^{15}$

On trouve là aussi une gradation depuis le signe donné seul (ex.1) jusqu'à la définition " étendue ${ }^{16}$ (ex.2) en passant par l'usage de termes métalinguistiques (" ça s'appelle ", «le nom c'est », etc.) :

Ex.1

$<$ P> c'est des plantes qui poussent dans l'eau ça a des grandes feuilles vertes et puis des feuilles blanches fleurs blanches jaunes ou roses c'est très joli

$<\mathrm{E}^{*}>$ c'est pas beau ça

$<\mathrm{P}>$ si c'est très joli

$<\mathrm{E}^{*}>$ ça s'appelle comment?

$<$ P $>$ nénuphars <elle écrit> et donc il a fait des grands tableaux je pourrais vous rapporter peut-être un livre pour vous montrer des grands tableaux même quelquefois qui font tout le tour d'un musée

Ex.2

$<\mathrm{P}>$ accueillant accueillant c'est le verbe accueillir

$<$ S1> ça veut dire quoi?

$<$ P> accueillir qu'est-ce que ça veut dire accueillir ? ce pays est très accueillant

$<\mathrm{S} 1>$ cher?

$<\mathrm{S} 2>$ très cher /

$<$ P $>$ non accueillant ça veut dire que qu'est-ce que ça veut dire accueillir?

$<\mathrm{N}>$ gentil

$<P>$ ouais c'est un peu ça c'est-à-dire accueillir ça veut dire / accueillir ça veut dire

$\mathrm{XX}$ par exemple moi/ euh quand je vous accueille on peut dire que je suis 
accueillante quand vous arrivez je vous dis bonjour avec un grand sourire par exemple hein c'est pas forcément moi mais je vous dis bonjour avec un grand sourire je vous fais entrer avec plaisir je vous fais entrer avec plaisir dans la classe d'accord ? ce pays est très accueillant ça veut dire que vous arrivez vous ou d'autres personnes arrivez dans un pays par exemple au Pakistan et au Pakistan ils sont très bien accueillis ça veut dire que les gens sont très gentils avec eux ils sont contents de les voir d'accord ? c'est ça accueillir / oui ? accueillant [...]

\subsection{L'intercompréhension ${ }^{17}$}

Dans la classe de langue, dans le cas de l'enseignement à un public hétérogène du point de vue linguistique, en contexte homoglotte, l'asymétrie entre l'enseignant et les élèves est renforcée par le manque de langue commune. Ainsi, tous les moyens sont bons pour comprendre et se faire comprendre. L'intercompréhension est donc d'après nous une pratique métalinguistique puisqu'il est question de faire passer du sens. Les énoncés produits comportent des mots métalinguistiques ( je ne comprends pas» (sousentendu : « ce que tu viens de dire », ex.1)), ou pas (« hein? », « quoi ? », fréquent chez les élèves, "pardon?», «comment?» chez les enseignants, ex.2) voire la simple répétition du mot ou de l'énoncé incompris suivi de l'intonation montante (ex.3) :

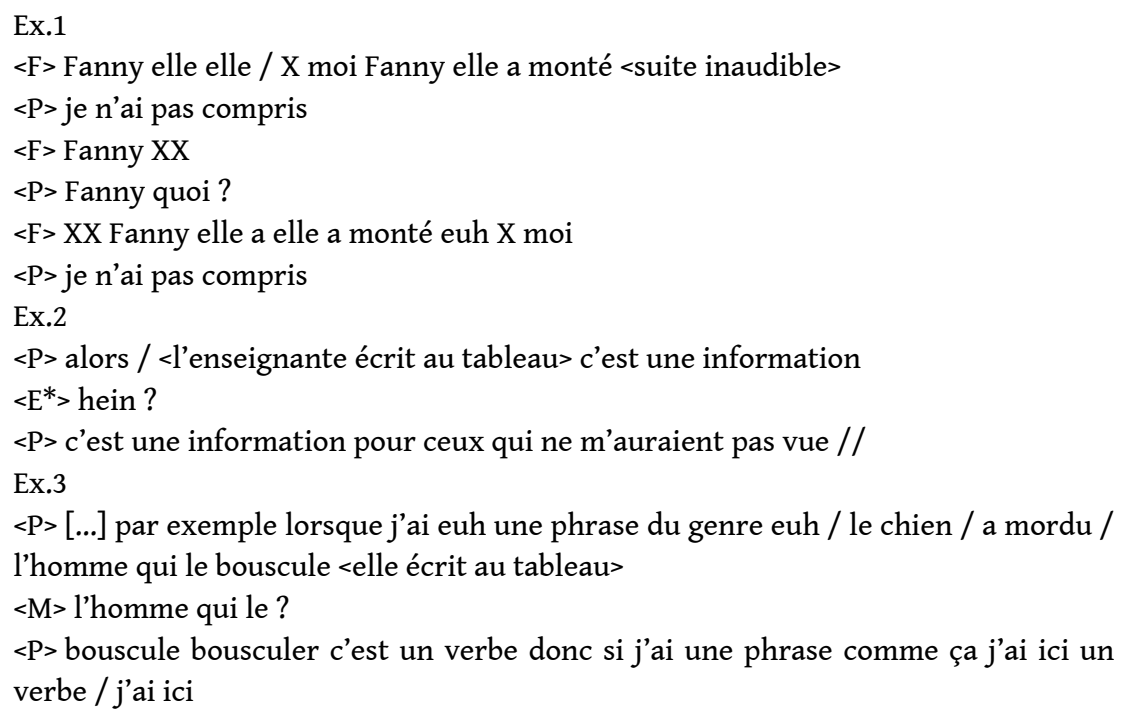

Dans les cas extrêmes, il arrive même que la langue ne soit pas du tout utilisée au profit de moyens que nous qualifions de "paralinguistiques " ( "à côté de la langue »), ce qui n'enlève rien à leur caractère métalinguistique. Il s'agit par exemple du mime, des gestes, de tout ce qui permet, surtout à l'élève, de se faire comprendre de l'enseignant quand «les mots manquent ». Bien sûr, le contexte fait que souvent les enseignants demandent de " parler ", parfois « en faisant des phrases complètes et correctes », et là, la métalangue reprend sa place.

Les moyens utilisés pour comprendre et se faire comprendre sont eux aussi très familiers dans les situations quotidiennes, y compris entre locuteurs parlant la même langue. C'est donc selon nous la pratique métalinguistique la moins typique de la classe de langue bien qu'elle soit beaucoup attestée dans notre corpus.

Les pratiques métalinguistiques, comme nous avons choisi de les appeler, sont nombreuses et prennent des formes variées. Elles se retrouvent toutes dans les classes de langue observées mais également au sein de discours standards. Bien que dans certains cas la terminologie métalinguistique soit complètement absente, il n'en 
demeure pas moins qu'on est face à du métalinguistique. Notre hypothèse (nous verrons plus loin si elle est vérifiée) est donc que plus on se dirige vers le moins spécifique à la classe, moins le lexique métalinguistique sera présent et inversement, la métalangue sera d'autant plus spécialisée que l'on sera dans le cadre de la description de l'organisation de la langue (en grammaire).

Dans la partie suivante nous allons nous attacher à décrire le lexique destiné à parler de la langue, à la décrire, à l'expliquer. Son hétérogénéité due à ses utilisations dans différents types de discours et contextes, nous a amenée à proposer une classification dont les frontières vont s'avérer poreuses.

\section{Classification du lexique métalinguistique ${ }^{18}$}

Pour classer le métalexique, nous avons choisi de nous baser sur les définitions lexicographiques des lexies relevées dans notre corpus (plus de 800, simples et composées ${ }^{19}$ ), utilisées par les enseignants mais aussi par les élèves pour parler de / sur la langue. Pour ce faire, nous nous sommes inspirée de Cuq (2001: 707), qui distingue les termes "essentiellement grammaticaux" (TEG) et les termes "non essentiellement grammaticaux » (NEG), qui composent le «métalangage grammatical». Les premiers sont ceux qui relèvent de la linguistique et de la didactique (subjonctif), les seconds sont les "termes d'usage plus courant", qui ont d'autres acceptions que purement grammaticale (article). Enfin, les termes non spécifiques (TNS) ont pour Cuq la même acception dans toutes les situations de communication (interrogation) ${ }^{20}$.

Pour nommer les catégories de lexies, nous nous sommes inspirée de Rey-Debove (1997: 26) qui classe le lexique d'une langue entre mots mondains " [...] destinés à parler de ce qui n'est pas le langage (le monde, les choses). Par exemple : maison, chaud, respirer, mortellement ", mots métalinguistiques "[...] destinés à parler du langage» et mots neutres "[...] qui s'intègrent indifféremment à l'un ou l'autre [des deux ensembles précédents] ».

31 Enfin, pour affiner notre classification, il a été nécessaire de prendre en compte la polysémie de beaucoup des lexies relevées dans notre corpus.

\subsection{Le métalexique " technique ${ }^{21}$ »}

Cette catégorie regroupe toutes les lexies dont aucune ne possède d'acception mondaine dans sa définition. Parmi celles que l'on retrouve chez tous les enseignants observés, c'est-à-dire y compris chez ceux qui travaillent auprès des plus jeunes, il y a par exemple : dire, lire, mot, répondre, texte ou encore verbe $e^{22}$.

33 La haute fréquence de ces lexies s'explique parce qu'elles ne sont pas typiques de la classe de langue. Par conséquent, les enseignants n'y reviennent pas nécessairement, les utilisant comme si elles étaient connues de tous. Or, nous avons pu constater que $m o t^{23}$ notamment, n'est pas forcément toujours compris par les élèves, en particulier quand il est mis en lien avec d'autres lexies:

$<\mathrm{P}>$ ben montre-le-moi sur le tien l'alphabet/ $\mathrm{U}$ donc tu vois tu as les quatre écritures du $\mathrm{U} /$ quatre écritures $\mathrm{du} \mathrm{U}$ <elle continue de vérifier les cahiers> vous soulignez bien dans le texte tous les mots où vous voyez $\mathrm{U} / /$ tous les mots où tu vois $\mathrm{U}$

$<\mathrm{E}^{*}>$ maitresse c'est quoi le mot $\mathrm{U}$ ? 

jeunes, texte est très souvent remplacé par histoire :

$<\mathrm{P}>[. .$.$] ce que vous vous allez faire vous allez souligner dans le texte dans l'histoire$ tous les mots soulignés je l'ai pas mis dans les consignes là-bas mais souligner / c'est? par exemple je vais souligner le mot Jules

40 - ou pour être paraphrasées :

$<P>$ c'est des clés parce que si c'était une clé il n'y aurait pas d'S mais comme il y en a plusieurs je vous avais déjà expliqué on met $<\mathrm{E}^{*}>$ des clés 
$<$ P $>$ un S quand y en a plusieurs un enfant il n'y a pas d'S plusieurs enfants il y a un $\mathrm{S}$ là les prénoms on met un $\mathrm{S}$ parce qu'il y a plusieurs prénoms d'accord ? clés [e] / et le dernier chut chut le mot

41 Cet évitement se justifie par au moins deux raisons dans les classes observées : l'âge des élèves et le niveau, en lien avec la progression. Un enseignant peut choisir de reporter à plus tard l'étude d'un point de langue, la terminologie associée étant évitée à un certain moment.

Bien sûr, les élèves utilisent aussi ces termes, parfois à bon escient, parfois non, en tout cas il est rare que ce soit de leur propre initiative. Et si les élèves contournent cette terminologie très particulière ou paraphrasent (terminaison remplacé par «à la fin »), faute de maitrise suffisante, il arrive aussi qu'ils fassent preuve de créativité en inventant une lexie pour remplacer celle qu'ils ne connaissent pas ou ont oubliée :

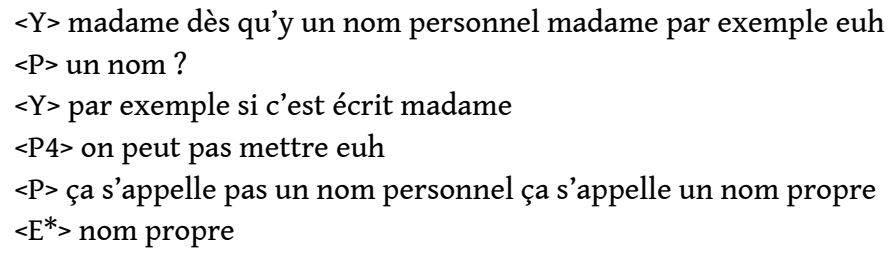

Dans ce cas, c'est un indice intéressant à prendre en considération pour analyser le rapport de l'élève à la métalangue, car cela permet de constater que l'élève a compris, seule lui manque la lexie "officielle». On peut donc constater son habileté métalinguistique, seuls lui manquent les «mots pour le dire», comme dans l'extrait suivant où l'élève évite synonyme :

$<\mathrm{E}^{*}>$ madame triste et monotone c'est la même?

Tous les métatermes de cette catégorie ne sont pas monosémiques. En effet, certains ont des acceptions mondaines. Ce sont ceux que nous avons décidé de dénommer «mixtes ».

\subsection{Le métalexique « mixte»}

Dans ce groupe nous retrouvons les lexies comportant au moins une acception technique et une autre mondaine dans leur définition (comme par exemple complément ).

On trouve deux cas de figure dans notre corpus : d'abord, celui où seule l'acception technique est attestée dans les propos (attribut, conjuguer....), sans doute parce que notre corpus ne sort pas de la salle de classe, ensuite celui où les deux types d'acception ont été relevés (complément, subordonnée...). Dans ce cas, il arrive que les lexies en question soient utilisées comme si elles étaient comprises de tous, malgré la confusion possible due à leur polysémie certainement parce que les deux acceptions sont imbriquées, non facilement discernables, et que le terme en question est là encore très courant dans les discours standards. C'est le cas par exemple de question, terme très répandu chez tous les enseignants sans exception. Ce qui est intéressant avec question, c'est que les enseignants utilisent ce terme en classe avec sa signification mondaine et le remplacent par interrogation pour parler de la forme des phrases dans le cadre d'une séquence destinée à l'étude de la langue. Nous pourrions faire l'hypothèse qu'il existe une certaine intuition chez ces enseignants concernant une différence à faire dans l'usage de la métalangue dès lors que le cours de grammaire a commencé. 

que font certains enseignants à l'acception mondaine (en principe connue des élèves du fait de son utilisation en dehors de la classe) pour expliquer le sens technique d'un terme. Ainsi complément, lexie très fréquente lors des cours de grammaire, est mise en lien avec compléter ( «le complément du nom complète le nom ») et le verbe est défini ("ça veut dire qu'on rajoute quelque chose »). L'exemple suivant en particulier est très intéressant pour illustrer notre propos ${ }^{24}$ :

$<P>$ alors les fonctions on va revoir là c'est la leçon d'aujourd'hui donc vous mettez la date et vous mettez les fonctions les fonctions du GN c'est la leçon d'aujourd'hui allez [...] alors qu'est-ce que c'est qu'une fonction ? / une fonction

$<\mathrm{E}^{*}>$ fonctionne madame

$<\mathrm{P}>$ qu'est-ce que c'est? / un emploi

$<\mathrm{E}^{*} 1>$ de travail

$<\mathrm{P}>$ un travail // [...] un même mot un mot ne change pas de nature [...] la nature c'est ce qu'on est donc ça c'est un verbe ça c'est un nom ça c'est un_ déterminant ça ne changera jamais

$<E^{*}>$ il changera jamais / jamais de la vie

$<P>$ ça peut pas changer / donc tu es un garçon tu resteras un garçon tu vas pas devenir un escargot / mais enfin normalement quand je te regarde je vois un garçon pas un escargot donc la nature ne change pas d'accord? / par contre la fonction change je suis une femme je ne vais pas me transformer en_ éléphant [...] la nature ne change pas / ma fonction elle change ici je suis professeur / donc une femme c'est une nature d'accord?

[...] la fonction ça peut être professeur d'accord? dans cette classe je suis professeur / quand je vais à V1 je suis formatrice [...] quand je suis au CIO [...] / quand je fais passer les tests / chut // je suis orientatrice [...] quand je suis euh je sais pas moi euh dans ma maison je suis maman /

$<\mathrm{E}^{*} 1>$ c'est la femme de ménage

$<\mathrm{P}>$ je suis entre autres femme de ménage oui ça aussi

Les deux autres termes mixtes avérés chez tous les enseignants observés, utilisés selon les cas avec uniquement leur acception mondaine ou avec les deux, sont temps et nom. Puisque ce sont des termes très répandus en dehors de la classe, ils ne font l'objet d'aucune élucidation sémantique. Avec les plus jeunes, temps n'est utilisé que dans son sens mondain, et la précision « du verbe » est souvent utilisée avec les plus âgés pour que cela soit plus précis. Nom est un cas particulier car les deux acceptions ${ }^{25}$ sont souvent attestées, et son statut est ambigu du fait de l'imbrication des significations mondaine et technique.

Quant aux élèves, ils en font un usage assez restreint, et comme pour les lexies techniques, c'est surtout en réponse au propos de l'enseignant, ou parce que certains termes étant répétés, ils utilisent les mêmes.

\subsection{Le lexique « mondain »}

50 Nous avons décidé de prendre en considération les lexies mondaines dans notre classification car elles peuvent prendre, en classe de langue, une dimension métalinguistique dans des usages tout particuliers, d'où l'hétérogénéité constatée.

51 C'est le cas par exemple de but, cause, conséquence, moyen, qui en elles-mêmes n'ont rien de métalinguistique, mais prennent une dimension métalinguistique du fait que complément de ou complément circonstanciel de sont sous-entendus. En fait, beaucoup de ces termes sont le résultat d'ellipses de lexies techniques composées. D'autres termes 
mondains prennent cette dimension métalinguistique parce qu'ils entrent dans le cadre d'une explication sur la langue (par exemple, condition ou hypothèse et même place).

D'autres termes mondains souvent relevés dans notre corpus, chez n'importe quel enseignant, sont ceux que l'on trouve dans les consignes métalinguistiques, visant à faire travailler les élèves sur la langue. Ce sont des mots à connaitre car ils appartiennent au français scolaire, et ils font aussi l'objet (plus systématiquement que les autres d'ailleurs), de précisions sémantiques. Ces termes sont notamment entourer, encadrer (on « encadre les verbes »), barrer, remplacer, souligner...

Enfin, une dernière série de termes répond à la volonté de simplifier le discours enseignant avec une utilisation métaphorique des termes mondains, qui n'est d'ailleurs pas propre aux classes de FLE/S. C'est le cas par exemple de morceau ( « les syllabes sont des petits morceaux de mots ", « l'extrait est un morceau de texte »).

En résumé, l'intérêt premier des lexies mondaines est leur capacité à pouvoir entrer et sortir de la sphère du métalinguistique. Elles peuvent revêtir une dimension métalinguistique voire technique, tout comme les mots métalinguistiques peuvent avoir perdu (en tout cas à première vue) leur caractère spécialisé du fait d'un usage important par ailleurs.

\section{Conclusion : pour aller un peu plus loin}

Nous pensons que cette classification, ainsi que les conclusions qui en découlent, pourraient avoir un intérêt didactique en ce qu'elles permettent une prise de conscience à la fois de l'hétérogénéité du métalexique et de la porosité des frontières. En effet, ce n'est pas parce qu'un terme est répandu dans la vie de tous les jours que son utilisation "va de soi » et qu'il sera vite compris et assimilé par un élève allophone. Même chose s'agissant des termes relevant de la "culture scolaire ", mondains certes, mais non forcément faciles d'accès. Inversement, la terminologie technique n'est pas nécessairement insurmontable, surtout si elle comporte des lexies mixtes dont les acceptions mondaines peuvent aider à la compréhension du sens technique ${ }^{26}$. La définition, pratique métalinguistique par excellence, omniprésente en classe de langue étrangère, aurait un rôle à jouer et pourrait ne pas être circonscrite au lexique mondain $^{27}$.

Deuxièmement, une autre question fondamentale, soulevée depuis longtemps, ressort ici, directement en rapport avec l'hétérogénéité : celle des choix au sein de cette métalangue pléthorique. Quels termes choisir et comment ? La métalangue ne pourraitelle être motivée sémantiquement, au moins dans un premier temps? Le fait de prendre conscience de choix à faire s'agissant de la terminologie grammaticale pourrait permettre une entrée en grammaire plus abordable, auprès d'un public allophone non nécessairement familier de la description du fonctionnement de la langue telle qu'elle est pratiquée en France (c'est-à-dire assortie de règles, d'une métalangue riche et complexe, d'exercices de mise en application, etc.).

$57 \mathrm{Si}$, en FLS, la réponse est fournie par l'institution scolaire grâce aux instructions officielles, qu'en est-il des autres situations d'enseignement/apprentissage telles que celle des étudiants d'échange inscrits dans les universités françaises ${ }^{28}$ ? Quelle métalangue utiliser dans ce cas et quelle utilisation ${ }^{29}$ en faire, sachant que le public est hétérogène à tous points de vue y compris celui de la «culture grammaticale»? 
Sachant aussi que le programme est très court, et que les cours de français doivent répondre à des exigences académiques très particulières (par exemple, rédiger une dissertation, ce qui demande des outils linguistiques bien particuliers)?

Le métalinguistique au sens large est, on l'a vu, omniprésent dans la classe de langue étrangère. Il apparait dans des pratiques, métalinguistiques, plus ou moins typiques de la classe, mais aussi dans les discours (celui des enseignants mais aussi celui des élèves). Cette omniprésence entraine une hétérogénéité qu'il est difficile de circonscrire.

Notre hypothèse n'est donc pas complètement vérifiée. En effet, les frontières sont perméables, le métalexique technique peut être présent en discours ordinaire ou bien contourné en classe de langue. À l'opposé, le lexique mondain peut devenir métalinguistique dans des pratiques et des discours propres à la classe. Par conséquent, il est important de ne pas réduire la métalangue à un vocabulaire spécifique, tout locuteur étant à même de s'exprimer sur sa (mé)connaissance de la langue sans pour autant connaître le jargon des spécialistes. Cela donne, comme nous avons pu le constater avec nos quelques exemples, des échanges riches, qui, ayant presque toujours la langue pour objet, sont métalinguistiques sans que des indices explicites (la métalangue) ne soient nécessairement attestés.

\section{BIBLIOGRAPHIE}

Cuq J.-P. (1996). Une introduction à la grammaire en français langue étrangère. Paris : Hatier/ Didier.

Cuq J.-P. (2001). Le métalangage grammatical aux niveaux débutant et intermédiaire dans quelques méthodes de FLE. In Colombat B. et Savelli M. (eds) Métalangage et terminologie linguistique : actes du colloque international de Grenoble. Louvain : Peeters. 705-724.

Masset A. (2009a). Enquête sur la métalangue dans l'enseignement/apprentissage du FLE/S à des élèves non francophones scolarisés en France, thèse de doctorat, Amiens.

Masset A. (2009b). La définition : un moyen d'accéder à la terminologie linguistique? Publif@rum 11 Autour de la définition.

Masset A. (2014). Lexique métalinguistique et classes de français langue étrangère/langue seconde en France. Le Discours et la langue 6.1 Métalangage et expression du sentiment linguistique profane.

Rey-Debove J. (1997). Le métalangage. Etude linguistique du discours sur le langage. Paris : Armand Colin (2 ème éd.).

Robert J.-M., Prescod P. (2014). La langue seconde à la croisée des chemins. Etudes de linguistique appliquée 174 Le français langue seconde, regards croisés. 139-146.

Trévise A. (1997). Métalexique, métadiscours et interactions métalinguistiques. LINX 36/1, Métadicours et enseignement/apprentissage des langues. 41-54. 


\section{NOTES}

1. En effet: «En didactique française, le terme langue étrangère définit une langue non maternelle, langue qui est apprise/enseignée à l'école (université, centre de langue, etc.), dans le pays de l'apprenant (si cette langue n'a pas de statut officiel) [...]. La langue seconde est, de son côté, toute langue non maternelle ou non initiale acquise par imprégnation sociale et/ou immersion scolaire, par exemple le français en cours d'apprentissage ou d'acquisition par des immigrés ou des enfants non francophones nouvellement arrivés en France » (Robert et Prescod 2014: 139).

2. Notre corpus se compose des transcriptions des enregistrements d'environ $120 \mathrm{~h}$ de cours de français auxquels nous avons assisté dans des CLIN (Classes d'Initiation) pour les élèves de l'école élémentaire, et des CLA (Classes d'Accueil) pour les élèves de collège, dans l'académie d'Amiens. Tous les exemples qui seront présentés au long de cet article sont extraits de ce corpus. Des entretiens avec les enseignants ont également été menés. Notons que depuis 2012 on ne parle plus de CLIN ou de CLA mais d'UPE2A (Unité Pédagogique pour Elèves Allophones Arrivants) pour nommer l'ensemble des différents types de classes accueillant ces élèves allophones.

3. C'est-à-dire la " grammaire ", nécessairement présente dans notre contexte (grammaire entendue ici dans le sens de morpho-syntaxe), mais aussi tous les moments consacrés au lexique ou à la phonétique.

4. Ce que nous avons observé parfois, sans cependant que les enseignants en aient toujours conscience. Par exemple, il arrive que certains repoussent à un moment ultérieur l'utilisation de telle ou telle lexie, ou alors qu'ils en définissent certaines en s'appuyant sur leur acception en discours ordinaire pour faire comprendre leur sens en grammaire (cf. 2.2. pour des exemples).

5. Cf. partie 2 .

6. Règle lui-même est d'ailleurs un terme très fréquent dans les classes observées.

7. «'Conceptualiser' c'est réfléchir sur le fonctionnement de la langue-cible. L'apprenant doit avoir la 'liberté d'exprimer comme il l'entend et par les moyens qu'il peut (langue maternelle, langue étrangère, schémas, dessins, etc.) la manière dont il comprend le fonctionnement des données de la langue étrangère qu'il est en train d'acquérir' [...]» (Besse, cité par Cuq (1996: 86)).

8. Rappelons que depuis de nombreuses années déjà, il est préconisé en FLE de faire de la grammaire de manière implicite, non décontextualisée, dans un but communicatif précis.

9. C'est ce qui est appelé grammaire implicite en didactique du FLE (que l'on oppose à la grammaire explicite).

10. $<\mathrm{P}>$ renvoie à l'enseignant, $<\mathrm{E}^{*}>$ à un élève non identifié, $<\mathrm{M}>$ à l'initiale d'un élève identifié. Quand plusieurs élèves répondent en même temps et sans que nous puissions les distinguer, nous notons les répliques audibles entre \{\} pour signaler que ce sont des réponses d'élèves différents. Dans le cas contraire, c'est-à-dire quand nous pouvons différencier les élèves, nous utilisons le soulignement traduisant le chevauchement. Le gras est utilisé pour l'insistance. /, // et /// signalent des pauses plus ou moins longues, [...] des passages supprimés, X, XX, XXX le nombre de syllabes inaudibles, _ un allongement de la syllabe et les transcriptions phonétiques sont entre crochets.

11. On voit d'ailleurs ici que l'enseignante corrige également directement, sans indice métalinguistique clair.

12. La séance porte en effet sur c'est un/ c'est une et ce sont des.

13. Ce classement des pratiques métalinguistiques de la plus spécifique à la classe de langue à la moins spécifique est sans doute discutable. Le but ici est avant tout de montrer leur hétérogénéité et leur présence dans les discours auxquels les élèves sont confrontés, y compris en dehors de la classe puisqu'ils sont en contexte d'immersion. 
14. Le recours à l'autonymie est très courant en classe, ce qui n'est pas sans poser de problèmes d'ambiguïté. C'est en tout cas un moyen très efficace pour contourner des difficultés comme par exemple celle liée à la terminologie.

15. Et de nouveau, avec traduire, un terme métalinguistique est utilisé.

16. Les définitions peuvent être morphologiques, paraphrastiques, par la situation (notre exemple), par inclusion, en extension, etc. (cf. Masset 2009b) et peuvent comporter plus ou moins de termes métalinguistiques.

17. Nous entendons "intercompréhension" dans le sens courant du terme, comme étant la capacité de compréhension mutuelle entre deux (ou plusieurs) interlocuteurs ou, comme ici, entre l'enseignant et les élèves.

18. Classification que nous reprenons de notre thèse de doctorat (Masset 2009a).

19. Les lexies composées sont des métatermes tels que par exemple adjectif indéfini, voix passive, connecteur logique. Elles sont très nombreuses et ont fait l'objet d'une description plus poussée dans notre thèse.

20. Nous avons cependant préféré élargir un peu cette conception de Cuq en raison de l'ambiguité du terme "grammatical ", d'autant plus que nous avions l'intuition qu'il fallait pouvoir englober d'autres types de lexies, celles qui n'avaient aucune signification grammaticale, linguistique ou didactique.

21. Nous utilisons cette étiquette faute de mieux pour rendre compte du fait que les lexies concernées n'ont que des définitions relevant du langage donc métalinguistiques.

22. Bien sûr, on sent bien une différence entre répondre ou mot et verbe, mais du point de vue de leur définition, chacune de ces lexies relève du métalinguistique uniquement.

23. Nous choisissons de nous pencher sur ce terme en raison de son omniprésence dans notre corpus et parce qu'il est également très utilisé en discours ordinaire.

24. On pourrait multiplier les exemples, notamment avec l'une des enseignantes de collège qui n'hésite pas à recourir aux sens mondains des lexies pour faire comprendre l'acception technique, et au-delà, le point de grammaire traité.

25. «Mot servant à désigner les êtres, les choses qui appartiennent à une même catégorie logique, et spécialt à une même espèce » vs "Une des parties du discours (-> catégorie), mot lexical qui désigne un individu, une classe » (Le Robert).

26. Cf. notre exemple sur la nature et la fonction des noms.

27. C'est ce que nous avons tenté de montrer dans notre article Masset (2009b).

28. En français sur objectifs universitaires, donc.

29. Auprès du public scolaire, même si la terminologie grammaticale officielle est évitée au début de l'apprentissage et/ou avec les plus jeunes, tôt ou tard elle fait son apparition, l'objectif étant que les élèves concernés intègrent au bout du compte les classes ordinaires, qui en font une utilisation massive.

\section{RÉSUMÉS}

A partir de l'analyse de discours tenus dans des classes destinées à accueillir des élèves allophones en France, nous voudrions montrer en quoi les pratiques et le lexique métalinguistiques sont omniprésents, hétérogènes et divers. Nous verrons qu'il existe, dans les classes de français langue seconde observées, un va-et-vient entre les lexies spécialisées, relevant 
de la linguistique et de la grammaire, et les mots des discours ordinaires, au sein de quelques pratiques métalinguistiques très représentées dans notre corpus.

From the analysis of speeches held in classes intended to accommodate non-native speakers pupils in France, I would like to show how metalinguistic practices and lexicon are ubiquitous, heterogeneous and diverse. I will analyse this phenomenon through French second-language classes observed in which there is a back-and-forth between specialized lexicons, connected to linguistics and grammar, and the words of ordinary speeches, within some recurrent metalinguistic practices represented in this corpus.

INDEX

Keywords : metalanguage, French as a Second Language, non-native speakers pupils.

Mots-clés : métalangage, Français Langue Seconde, élèves allophones.

\section{AUTEUR}

\section{ANGÉLIQUE MASSET-MARTIN}

Grammatica, Université d'Artois 